\title{
Tuning STEM: Tailoring the Incident Probe, Scattering Dynamics and Detector Geometry for Maximum Specimen Information
}

\author{
J. Etheridge ${ }^{1,2}$, W. Chao ${ }^{2}$, E. Drath Bøjesen ${ }^{1}$, Y.Guo $^{2}$, M. Krajnak ${ }^{2}$, W. Li ${ }^{2}$, CL. Zheng ${ }^{1}$ \\ ${ }^{1 .}$ Monash Centre for Electron Microscopy, Monash University, VIC 3800, Australia \\ 2. Department of Materials Science and Engineering, Monash University, VIC 3800, Australia
}

In the simplest sense, a transmission electron scattering experiment comprises three components; the incident electron wave field; the scattering within the specimen and the exit electron wave field. In a scanning transmission electron microscope (STEM), we have some degree of control over these three components, via the probe-forming optics, specimen orientation and detector geometry. These give us the ability to tune, within certain limits, each component of the experiment to target and extract specific types of specimen information. This is the essence of a S/TEM experiment.

Recent developments in electron-optical elements, from aberration-correctors to diffraction gratings, are providing unprecedented control over the incident wave field, enabling new structured waveforms such as Bessel and vortex beams to be generated, for example [1-4], as well as improving the fidelity of conventional spherical and planar waveforms. At the other end of the experiment, the advent of fast detectors that allow the full exit wave intensity distribution to be recorded for each probe position, are providing unprecedented control over the detection of the exit wave intensity distribution and revolutionizing the information that can be obtained.

In the 'middle' of the experiment, we have some limited control over the scattering dynamics, by controlling the specimen orientation with respect to the incident wave field, for example [5].

In this paper, we tune the incident wave form (for example [6]) and specimen orientation to tailor the scattering within the specimen in order to target and generate specific information about the specimen. We then harness this information with matching detector geometry.

Using a double-aberration-corrected FEI Titan ${ }^{3}$ 80-300 FEG-TEM fitted with a pixelated detector (the "electron microscope pixel array detector" EMPAD based on the Cornell design [7]) and a high-speed CMOS-based CETA camera, we illustrate this approach with practical examples, including applications to measuring composition in III-V semiconductors, structural modulations in functional perovskites and surface alloys on metallic nanoparticles. 
References:

[1] M Uchida and A Tonomura, Nature 464 (2010) p. 737.

[2] J. Verbeeck, H. Tian and P. Schattschneider, Nature 467 (2010) p. 301.

[3] BJ McMorran et al., Science 331 (2011) p. 192.

[4] CL Zheng, TC Petersen, H Kirmse, W Neumann, MF Morgan and J Etheridge, Phys Rev Lett 119 (2017) 174801.

[5] PH Nakashima, AF Moodie and J Etheridge, PNAS 110 (2013) p. 14144.

[6] DT Nguyen, S.D. Findlay and J Etheridge, Ultramicroscopy 184 (2018) p. 143.

[7] MW Tate et. al., Microscopy and Microanalysis 22 (2016) p. 237.

[8] The authors acknowledge funding from the Australian Research Council grants DP150104483, DP160104679 and LE0454166. 